\title{
Concise Commentary: Caution, Not Cauterization-Management of Post-ESD Colorectal Hemorrhage
}

\author{
Yoshii Shinji $^{1}$
}

Published online: 13 November 2018

○) Springer Science+Business Media, LLC, part of Springer Nature 2018

Colorectal endoscopic submucosal dissection (ESD) is gaining recognition as a safe and minimally invasive procedure to remove early-stage colorectal cancer. Since colorectal ESD is often complicated by delayed bleeding in 0.5-9.5\% patients, it is important for endoscopists to determine when emergency endoscopy is necessary in order to treat this complication [1]. Although massive bleeding generally requires initial endoscopic hemostasis, most cases of bleeding do not require emergency repeat colonoscopy since bleeding usually spontaneously stops within several hours and does not recur prior to hospital discharge [2]. Nevertheless, no consensus exists regarding the safe and appropriate management of delayed bleeding following ESD.

To address this question, Chiba et al. [3] in the current issue of Digestive Diseases and Sciences reported a retrospective study assessing the feasibility of the "watch and wait" strategy, whereby clinicians based their decision to carry out emergency endoscopy on the basis of the patients' vital status and symptoms. This strategy was used in cases of delayed bleeding after colorectal ESD when the patient had a shock index less than one and/or less than five occurrences of moderate hematochezia. Such patients, carefully observed throughout their clinical courses, underwent no emergency colonoscopy. Over 400 patients were recruited in the study, divided into a bleeding group (27 patients) and a non-bleeding group. Since none of the patients in the bleeding group required emergency endoscopy to treat their delayed bleeding, the authors concluded that the "watch and wait" strategy for bleeding following colorectal ESD is safe.

Complications such as perforation and bleeding during and after colorectal ESD can be serious and life threatening. In this regard, Chiba et al. discussed several reasons why the necessity for emergency endoscopic treatment is

Yoshii Shinji

shinji-yoshii@umin.ac.jp

1 Department of Gastroenterology, Sapporo Medical Center, NTT EC, S1 W15, Chuo-ku, Sapporo 060-0061, Japan less common after colorectal ESD than after gastric ESD. First, the stomach has much larger caliber submucosal blood vessels than does the colon, conferring a higher risk of massive delayed bleeding after gastric ESD that in turn can be complicated by hemodynamic compromise and aspiration pneumonia. In the case of colorectal ESD, most delayed bleeding stops spontaneously due to the smaller diameter vasculature, with a lessened risk of morbid complications. More emphasis should also be placed on delayed perforation as a serious post-procedural complication of colonic ESD since consequent bacterial peritonitis and may require surgical intervention. Thus, determining the need for endoscopic coagulation procedures is of utmost importance.

To elucidate the risk factors for delayed bleeding and assess whether emergency colonoscopy is necessary based using the "watch and wait" strategy, Chiba et al. investigated patient-related factors and lesion-related factors. Multivariate analysis revealed that the risk factors for bleeding were a rectal lesion size $\geq 40 \mathrm{~mm}$ with a higher overall risk of rectal ESD for delayed bleeding, perhaps since defecationrelated straining exerts greater mechanical shear force on the richly vascularized rectal venous plexus. The study also suggested that larger lesion size was a risk factor for delayed bleeding, corroborating a previous nationwide study [4]. As the resection size gets larger, endoscopists are required to carry out more complicated operations, resulting in longer procedure times. As mentioned by Chiba et al., the incidence of delayed bleeding may depend on the skill level needed for the procedure, which is difficult to objectively evaluate. To overcome this problem, authors of a previous study stated that pre-procedural training is needed for all practitioners performing colorectal ESD [5].

Although Chiba et al's study incorporated over 400 patients, only 27 patients with delayed bleeding were followed using the "watch and wait" strategy. Since no subject required emergency endoscopy, the study had no control arm, nullifying the assessment of risk factors for emergency colonoscopy to treat delayed bleeding. One previous 
publication involving a larger cohort reported that delayed bleeding was observed in $2.2 \%$ of patients after colorectal ESD [4]. Therefore, a future multicenter study with a larger cohort is necessary to confirm the observations of Chiba et al. and to better analyze the risk factors for colorectal ESD complications.

As the incidence of colorectal cancer is rising, so is the demand for effective and minimally invasive therapy. In the aging population in particular, minimally invasive procedures are preferable, since such patients often have comorbidities such as diabetes, hypertension, and vascular diseases that increase procedural risk. The study by Chiba et al. provides data supporting the safety of the "watch and wait" strategy following colorectal ESD.

\section{References}

1. Tanaka S, Terasaki M, Hayashi N, Oka S, Chayama K. Warning for unprincipled colorectal endoscopic submucosal dissection: accurate diagnosis and reasonable treatment strategy. Dig Endosc. 2013;25:107-116. https://doi.org/10.1111/den.12016.

2. Ogasawara N, Yoshimine T, Noda H, et al. Clinical risk factors for delayed bleeding after endoscopic submucosal dissection for colorectal tumors in Japanese patients. Eur J Gastroenterol Hepatol. 2016;28:1407-1414. https://doi.org/10.1097/MEG.0000000000 000723 .

3. Chiba H, Ohata K, Tachikawa J, et al. Delayed bleeding after colorectal endoscopic submucosal dissection: when is emergency colonoscopy needed? Dig Dis Sci. https://doi.org/10.1007/s1062 0-018-5310-2. [Epub ahead of print].

4. Nakajima T, Saito Y, Tanaka S, et al. Current status of endoscopic resection strategy for large, early colorectal neoplasia in Japan. Surg Endosc Interv Tech. 2013;27:3262-3270. https://doi. org/10.1007/s00464-013-2903-x.

5. Ohata K, Ito T, Chiba H, Tsuji Y, Matsuhashi N. Effective training system in colorectal endoscopic submucosal dissection. Dig Endosc. 2012;24:84-89. https://doi.org/10.111 $1 / \mathrm{j} .1443-1661.2012 .01272 . x$. 